\title{
The offshore spill oil recovery monitoring system based on the wireless communication
}

\author{
Wen Dai ${ }^{1, a}$, XuYing Ren ${ }^{1, b}$ and Qian $\mathrm{Li}^{1, \mathrm{c}}$ \\ ${ }^{1}$ College of Mechanical and Electrical Engineering, Shandong University of Science and Technology, \\ Qingdao, China \\ asdwust@163.com, bjirenxuying@163.com, Cliqian19870726@163.com
}

Keywords: spill oil recovery, MCU, data acquisition, wireless communication

\begin{abstract}
This paper adopted the dynamic inclined plan (DIP) oil skimmer. The monitoring system composed of oil skimmer and workstation. The workstation on the vessel monitored the oil sump tank's oil level in real-time. And the MCU,controlled the oil pump to suck spilled oil. In the system's design, data transmission between the oil skimmer and the workstation is one of the important factors that influence the systems' accuracy. This paper proposes the wireless communication method. And the wireless transceiver nRF24L01 chip composes the wireless transmission system. Experiments show that this scheme overcomes the inherent corrosion problem in the seawater and improves the system's reliability.
\end{abstract}

\section{Introduction}

With the rapid development of the global economy, there are more and more demanding for oil resources around the globe, the scope of oil drilling and transportation in offshore area has been growing. As a consequence, spill oil accidents at sea are increasing. These accidents have brought great losses to fishery, breeding, travel industry and marine economy industry, and causing serious pollutions to the marine environment and the coastlines, posing huge threat to the marine ecological resources [1]. To dealing with all kinds of spill oil accident, spill oil recovery monitoring system plays an important role.

In traditional spill oil recovery system, wired transmission like RS-485 bus and CAN bus is commonly adopted. However, cable links are required between the workstation and the sensor. This wired deployment makes the whole system much more complex. Moreover, water corrosion as well as the adverse environment will reduce the reliability of the cable transmission. Due to the above reasons, rather than using traditional wired communication, wireless communication technology is adopted to acquire the sensor data. This will not only overcome the complexity of cable deployment under sea, but also improve the reliability of the signal transmission.

\section{The composition of the spill oil recovery and monitoring system}

Spill oil recovery device. At present, brush type, vacuum type and the disk type oil spill recovery processing methods are commonly used. But the above methods are all hard to finish the recovery in a short time especially for some large-scale spill oil recovery. This paper adopts vessel's holding spill oil recovery integrated system. And the integrated system consists of vessel, fences and oil skimmer, shown in fig. 1.

The dynamic cant plan (DIP) oil skimmer is made up of oil sump tank and inclined belt, as is shown in fig. 2. In the process of recovering the spill oil, the inclined belt produces the traction to guide the oil-water layer overflowing along with the rotation direction. The water layer goes down and the spill oil layer goes up to the top of the oil sump tank. Real-time detection system is used to detect the oil height in the tank. When the oil layer achieves a certain thickness, the oil pump begins to suck the oil. When oil layer up to the top limit, the system will control the speed of the inclined belt, slows down the speed of sucking the spill oil, and thereby improves the recovery efficiency of the system [2]. 

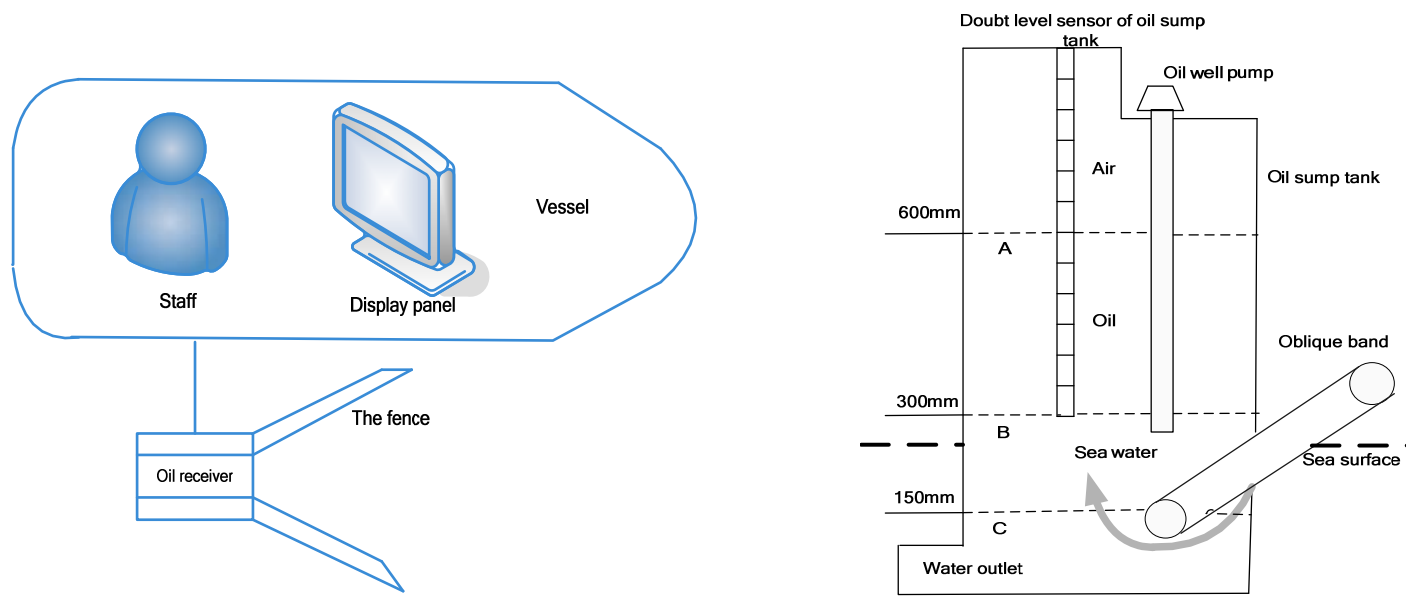

Fig. 1 Spill oil recovery device Fig. 2 The calibration of the oil tank and the liquid level

The system hardware structure. The overall hardware circuit structure of the spill oil recovery and monitoring system is shown in fig. 3 . The whole system is constituted by the oil skimmer and the workstation, seen in the dotted portion of the figure.

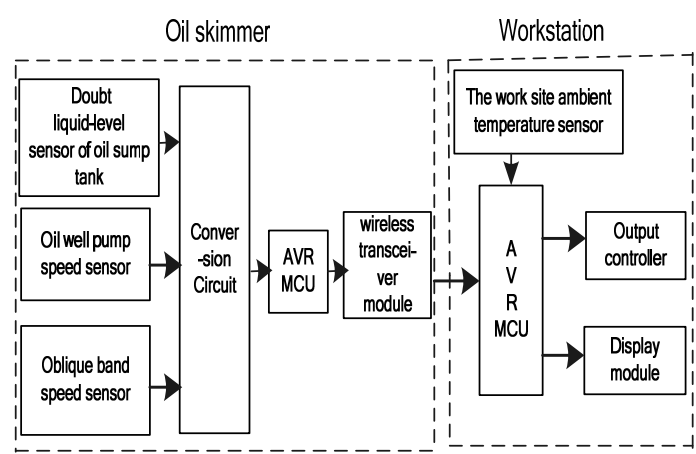

Fig. 3 The overall hardware circuit structure

The oil sump tank level's measurement adopts the double liquid-level sensor. The speed measurement of the reel and the oil pump adopts Hall sensor. And the environmental temperature's measurement adopts the temperature sensor. Through the signal converting circuits, the above signals are transmitted to the MCU for data processing and displaying. The data transmission between the oil skimmer and the workstation adopts wireless communication. Through the software filter, digital signal linear processing, the above data are sent out through the wireless module nRF2401. The data access system, consisted by MCU and wireless module in the workstation, will process and further display the received data [3].

\section{The oil sump tank's level monitoring}

The double level data acquisition of the oil sump tank. In the working process of the dynamic cant plan (DIP) oil skimmer, the oil sump tank contains three mediums: the upper air, the middle-level oil and the lower water. The oil skimmer's goal is: eliminating the air, sucking off the oil and cleaning off the water. In order to achieve this goal, with the aid of multiple-interface-detection and system-control technology, the system adopts the automatic separation scheme to finish the recovering work.

The data collection of the oil sump tank's double-liquid level has shown in fig. 2. In the data acquisition process, when the oil level is higher than the lower limit (about $300 \mathrm{~mm}$ ), the oil pump starts and then begins to suck the oil. Conversely, when the oil level is below the lower limit (about $150 \mathrm{~mm}$ ), the oil pump deduces the speed and stops working. So the oil lower-limit point and the water upper-limit point are the data collections' alarm points. 
Based on the above requirements, the system chooses SDJML-700 oil-water double-level measurement instrument to measure the oil sump tank's level. SDJML-700 oil-water double-level measurement instrument can not only measure single medium level, but also can accurately measure levels of two different medium layers within the oil tank, such as oil level and water level. SDJML-700 oil-water doubt level measurement instrument's two ways current output signals ( $4 \sim 20$ $\mathrm{mA}$ ) need to be converted into digital signals that AT90SMEG103 MCU can be identified. The transform circuit is shown in fig. 4 . The $4 \sim 20 \mathrm{~mA}$ current signals connects $250 \Omega$ sampling resistance, and then the current signals are converted into $1 \sim 5 \mathrm{~V}$ voltage signals. After the buffer isolation of the voltage followed instrument and the $\mathrm{RC}$ low pass filtering processes, current signals finally completes the conversion of analogues into digital amounts. Among them, the water and oil ports are separately connected with ports PF2 (ADC2) and PF1 (ADC1) of the MCU [4].

Measurement of the inclined belt speed. The system adopts Hall sensor to measure the speed of the inclined belt drum and the oil-well pump. Three ways are commonly used to measure the speed. They are: frequency method, cycle method and frequency/cycle method. Because the spill oil recovery and monitoring system's rolling speed ranges from 40 to $100 \mathrm{r} / \mathrm{min}$, so the system adopts the cycle method. Speed values' calculation is shown in Eq. 1:

$$
n=\frac{60 \bullet f}{p} \cdot \frac{1}{m}
$$

In the formula, $f$ stands for clock pulse frequency; $p$ stands for the number of sensor's pulse; $m$ stands for the number of the clock pulse.

The application of wireless communication in the system. Vessel holds the oil spill recovery system works on the sea. In the process of data collection communication, the wire transmission will cause series of problems, such as the tedious installation, the cable connection and the sea water corrosion. All these factors are fault hidden dangers that will affect spill oil recovery system's emergency and sustainability. Therefore, the spill oil recovery and monitoring system chose the wireless transmission. The sensors collect the signals and then sent them to the operation of the accept tanker for data monitoring and controlling. Because the system's power is quite low and the communication distance is very short, the system adopts the single-chip RF transceiver chip nRF24L01 to complete the data's wireless transmission.

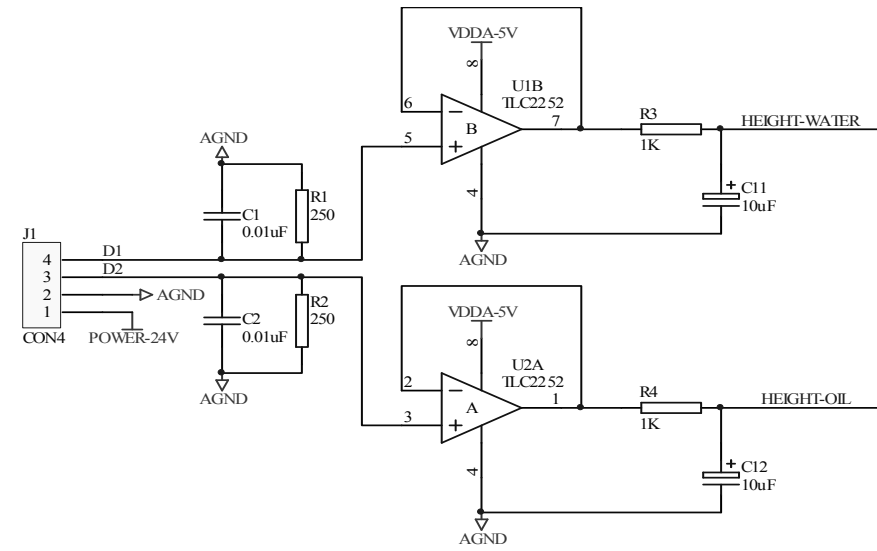

Fig. 4 The transform circuit of the oil sump tank level

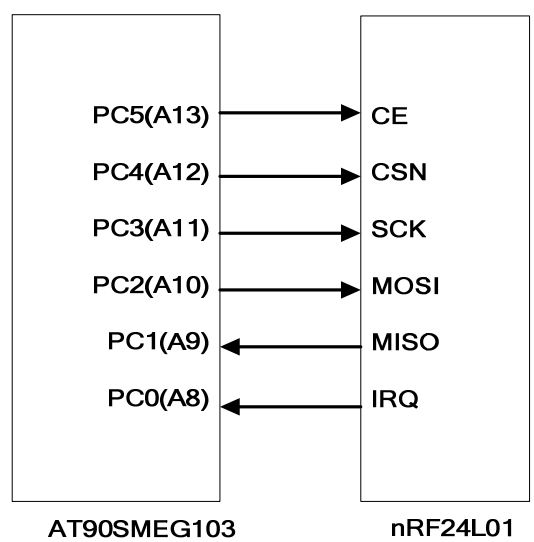

Fig. 5 Hardware interface circuit

The hardware interface connection circuit between NRF24L01 wireless RF transceiver module and AT90SMEG103 MCU are shown in fig. 5. NRF24L01's CE, CSN, SCK, MOSI, MISO, IRQ ports are linked to AT90SMEG103 MCU's PC5 PC0 mouths to control nRF24L01's way of working [5]. AT90SMEG103 MCU's working voltage is $5 \mathrm{~V}$ and the drive current is very large, but nRF24L01 module's working voltage is only $3.3 \mathrm{~V}$. In order to reduce the voltage and limit the current, cascade a resistance of $5.1 \Omega$ between them can act as a protection.

The software process of the wireless communication transceiver. AT90SMEG103 MCU controls the nRF24L01 chip's working process. It mainly includes the initialization configuration of 
the NRF24L01, and then sending/receiving the data and finally storing the data. And the specific procedure is shown in fig. 6.
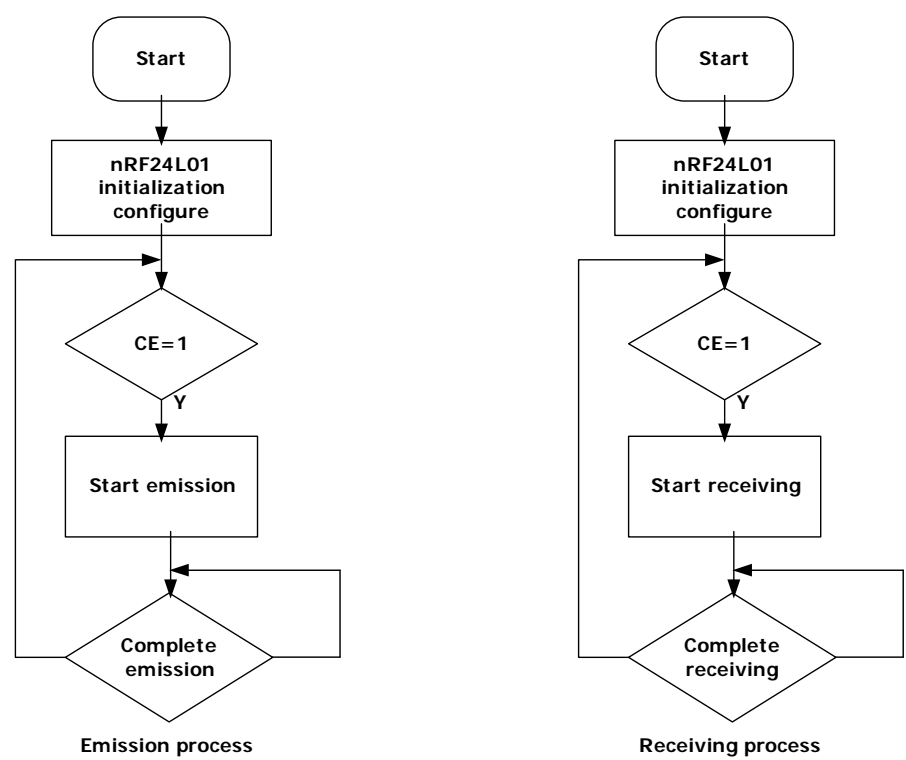

Fig. 6 The software process of the wireless communication transceiver

\section{Conclusions}

The offshore spill oil recovery monitoring system adopts wireless communication to transmit the data between the workstation and oil skimmer. According to the test of the date transmission ability, the system is reliable. Experiment results showed that the major factors that influence the wireless communication are the application environment, the transmitter's output and receiver's sensitivity. In order to improve the accuracy of this system shielding measures and necessary filtering networks should be considered.

\section{References}

[1] Woolga L: Assessing the Increasing Risk of Marine Oil Pollution Spills in China. Proceedings of International Oil Spill Conference (2008), p.711-715

[2] Zhang Dewen Zhang Tongmao: Whole Hydraulically Driving High-efficiency Spilled Oil Disposing System. Reserch on waterborne transportation (2010), P.18-22

[3] ShiZhijun,CaiJianping: A new kind of high speed wireless RF thansceiver-Nrf24L01 and its application. International Electronic Elements (2007), P. 42-44

[4] GaoJing, ZuoJinPeng: the hardware design of a study board based on STC-MCU. Electronic technology(2009), P. 8-10

[5] Li Hui, SongShi and Zou Jianjiang: Design of wireless date transceiver system based on ARM\&Nrf24L01. Foreign electronic components (2008), P. 44-46 